Bull. Austral. Math. Soc.

VoL. 59 (1999) [65-81]

\title{
TWO AMALGAMS RELATED TO THE ALTERNATING GROUP ON SIX LETTERS
}

\section{Panagiotis Papadopoulos}

A characterisation is given of some of the parabolics found in $\mathrm{Co}_{3}$, and $\mathrm{SP}_{4}$ (9) using the amalgam method.

\section{INTRODUCTION}

Leg $G$ be a finite group, $p$ a prime, $S \in \operatorname{Syl}_{p}(G)$ and $B=N_{G}(S)$. A proper subgroup of $G$ which contains a conjugate of $B$ is called a parabolic subgroup of $G$. The set $\mathfrak{I}$ of parabolic subgroups of $G$ ordered by inclusion becomes a partially ordered set called the parabolic geometry of $G$. In recent years the parabolic geometry (in particular for $p=2$ ) has been used to study, construct, characterise and prove uniqueness of many of the sporadic finite simple groups. The parabolic geometries (again for $p=2$ ) also play an important role in the ongoing revision of the classification of the finite simple groups, in particular in the so called quasi-thin and uniqueness cases.

Parabolic subgroups have been studied most intensively for $p=2$ but many interesting examples exist (besides the groups of Lie type) for arbitrary primes.

Recall that $G$ is an amalgamated product of $P_{1}$ and $P_{2}$ if $\left(G, P_{1}, P_{2}\right)$ has the following properties:

(i) $P_{1}$ and $P_{2}$ are finite subgroups of $G$.

(ii) $G=\left\langle P_{1}, P_{2}\right\rangle$.

(iii) Let $S \in \operatorname{Syl}_{p}\left(P_{1} \cap P_{2}\right)$ and $B=N_{P_{1} \cap P_{2}}(S)$; then $B=N_{P_{i}}(S), i=1,2$. In particular $S \in \operatorname{Syl}_{p}\left(P_{i}\right), i=1,2$.

(iv) No nontrivial normal subgroup of $G$ is contained in $B$.

To any amalgamated product $\left(G, P_{1}, P_{2}\right)$ we can associate a graph $\Gamma$ whose vertices are the cosets of $P_{1}$ and $P_{2}$ in $G$ and two cosets are adjacent if they are distinct and have non-empty intersection We remark that if $B=N_{G}(S)$ then the graph $\Gamma$ can be embedded into the parabolic geometry of $G$.

The amalgamation method has proven very successful in determining the structure of $P_{1}$ and $P_{2}$ assuming the action of $P_{1}$ and $P_{2}$ on their neighbours $\Delta\left(P_{1}\right)$ and $\Delta\left(P_{2}\right)$ respectively in the graph $\Gamma$ is given.

Received 27th May, 1998

Copyright Clearance Centre, Inc. Serial-fee code: 0004-9729/99 \$A2.00+0.00. 
Let us assume for simplicity that $P_{1} \cap P_{2}=B$. Let $Q_{i}=O_{p}\left(P_{i}\right), L_{i}=O^{p}\left(P_{i}\right)=$ $\left\langle S^{P_{i}}\right\rangle$ and $P_{i}^{(l)} / Q_{i}=C_{G_{i} / Q_{i}}\left(L_{i} / Q_{i}\right)$. Then it is easy to see that $P_{i}^{(l)}$ is precisely the kernel of the action of $P_{i}$ on $\Delta\left(P_{i}\right)$ and $L_{i}$ acts transitively on $\Delta\left(P_{i}\right)$. Hence the group $L_{i} / Q_{i}$ carries most of the information about the action of $P_{i}$ and $\Delta\left(P_{i}\right)$ and we refer to the pair $\left(L_{1} / Q_{1}, L_{2} / Q_{2}\right)$ as the type of the amalgamated product $\left(G, P_{1}, P_{2}\right)$.

The main task of the amalgam method can now be described as determining $\left(P_{1}, P_{2}\right)$ from the type $\left(L_{1} / Q_{1}, L_{2} / Q_{2}\right)$. For example, in [9] we determined the structure of $\left(P_{1}, P_{2}\right)$ of type $\left(\Theta, \mathrm{SL}_{2}(3)\right)$, where $\Theta \cong \mathrm{PSL}_{2}(9), M_{11}, M_{12}$ or $2 \cdot M_{12}$, for $p=3$.

For the remainder of this paper we shall work under the following hypothesis:

$\left(^{*}\right)\left(G, P_{1}, P_{2}\right)$ is an amalgamated product of type $(\Theta, \Psi)$ for $p=3$ so that:

(i) $\Theta \cong \mathrm{PSL}_{2}(9), M_{11}, M_{12}$ or $2 \cdot M_{12}$,

(ii) $\Psi \cong \mathrm{PSL}_{2}$ (9) or $\mathrm{SL}_{2}(9)$,

(iii) $C_{P_{i}}\left(O_{3}\left(P_{i}\right)\right) \leqslant O_{3}\left(P_{i}\right)$ for $i=1,2$.

Introduce now the following notation: $G \sim 3^{d_{1}+\ldots+d_{n}} H$ means that there exists a normal series

$$
1=H_{0} \leqslant H_{1} \leqslant \ldots \leqslant H_{n} \leqslant G
$$

so that for $i=1,2, \ldots, n, H_{i} / H_{i-1}$ is an elementary Abelian minimal normal subgroup of $G / H_{i-1}$ with $\left|H_{i} / H_{i-1}\right|=3^{d_{i}}$ and $G / H_{n} \cong H$.

Also, by $G \sim 2 \cdot H$ we mean that $G / Z(G) \cong H,|Z(G)|=2$ and $Z(H) \leqslant H^{\prime}$.

We are now able to state our main result.

TheOREM. Under hypothesis $\left(^{*}\right)$ the possible pairs $\left(L_{1}, L_{2}\right)$ are:

(i) $\left(3^{5} M_{11}, 3^{1+4} \mathrm{SL}_{2}(9)\right)$,

(ii) $\left(3^{6} \mathrm{PSL}_{2}(9), 3^{1+1+4} \mathrm{SL}_{2}(9)\right)$.

Note that the above examples can be found in $G \cong \mathrm{Co}_{3}$ and $\mathrm{PSP}_{4}(9)$ respectively.

\section{Properties of $\Theta, \Psi$ and their Modules - THE GRAPH $\Gamma$}

A Steiner system $S(l, m, n)$ is a pair $(\Omega, \mathcal{B})$, where $\Omega$ is a set of size $n, \mathcal{B}$ is a set of subsets of size $m$ called blocks and such that every subset of size $l$ in $\Omega$ lies in a unique member of $\mathcal{B}$.

By [10], there exists, up to isomorphism, a unique Steiner system of type $S(5,6,12)$. Let $\mathcal{S}=S(5,6,12)$. Define then the Mathieu group on 12 points to be the group $M_{12}=\operatorname{Aut}(\mathcal{S})=\left\{\pi \in \operatorname{Sym}(12) \mid B^{\pi}\right.$ is a block for all blocks $\left.B\right\}$.

Define $M_{11}$ to be the stabiliser of a point in $M_{12}$. Then $M_{11}$ is 4-transitive on eleven points and its corresponding Steiner system is $S(4,5,11)$. 
LEMMA 2.1.

(a) $\left|M_{12}\right|=12 \cdot 11 \cdot 10 \cdot 9 \cdot 8=2^{6} \cdot 3^{3} \cdot 5 \cdot 11$.

(b) $M_{12}$ has two classes of involutions, say $D_{1}$ and $D_{2}$. Moreover if $D_{1}=$ $\{x \mid x$ fixes a point $\}$, then $x \in D_{1}$,

if and only if $x$ fixes a point

if and only if $x$ fixes four points

if and only if $x$ belongs to a normaliser of a Sylow 3-subgroups of $M_{12}$ if and only if $x$ lifts to an involution in $2 \cdot M_{12}$.

Proof: See [1] and [6].

NOTATION 2.2. To avoid repetitions we shall use the following notation throughout:

$$
X \cong(2) H \text { means that either } X \cong 2 \cdot H \text { or } X \cong H \text {. }
$$

DEFINTION 2.3: Let $X$ be a finite group. Slightly abusing the standard defintion we shall say that $X$ is 3-stable provided that the following condition holds: If $V$ is an irreducible $G F(3) X$-module and $A \leqslant X$ is such that $[V, A, A]=1$ then $[V, A]=1$.

Lemma 2.4. Let $Y$ be a finite group. Then:

(a) The following statement is equivalent to $Y$ being 3-stable: let $V$ be any $G F(3)$-module and $A \leqslant Y$ with $[V, A, A]=1$. Then $A C_{Y}(V) / C_{Y}(V) \leqslant$ $\mathrm{O}_{3}\left(Y / C_{Y}(V)\right)$.

(b) $Y$ is 3-stable if and only if $Y / O_{3}(Y)$ is 3-stable.

(c) If every element of order 3 in $Y$ lies in a perfect simple 3-stable subgroup of $Y$ then $Y$ is 3-stable.

Proof: See [9].

Definition 2.5: A GF(3) $X$-module $V$ is called an $F F$-module for $X$ if $C_{X}(V)=1$ and if there exists a non-identity 3-subgroup $A$ of $X$ such that $|V| /\left|C_{V}(A)\right|$ $\leqslant|A|$.

Lemma 2.6. $\Theta$ is 3-stable; in particular $\Theta$ does not have an $F F$-module.

Proof: The proof that $\Theta$ is 3-stable can be found in [9]; Thompson's Replacement Theorem $[5,8.2 .5]$ implies that a group with an irreducible $F F$-module is not 3-stable and hence $\Theta$ does not have an $F F$-module.

Definition 2.7: Let $X \cong \mathrm{SL}_{2}$ (9) and let $W$ be a faithful $G F(3) X$-module. Then $W$ is called a natural $\mathrm{SL}_{2}(9)$-module for $X$ if $W$ carries the structure of a 2-dimensional vector space over $G F(9)$ invariant under the action of $X$. 
It is worth mentioning at this point that

$$
A_{6} \cong \mathrm{PSL}_{2}(9) \text { and } 2 \cdot A_{6} \cong \mathrm{SL}_{2}(9)
$$

REMARK 2.8 .

(i) $\mathrm{PSL}_{2}(9)$ has four irreducible $G F(3)$-modules; their dimensions are: 1, 4, 6 and 9.

(ii) Let $X=\mathrm{SL}_{2}(9)$ and let $V$ be an $F F$-module. Then

$$
V=[V, Z(X)] \oplus C_{V}(X)
$$

and $[V, Z(X)]$ is a natural $\mathrm{SL}_{2}(9)$-module. [8, p.469 and 470$]$.

(iii) $\quad M_{11}$ has two non-trivial irreducible $G F(3)$-modules of dimension less than or equal to 8 ; moreover, both have dimension five and they are dual to each other [7].

Definition 2.9: Let $\Gamma=\left\{P_{i} x \mid x \in G, i=1,2\right\}$. From now on, small Greek letters will always denote elements of $\Gamma$. Make $\Gamma$ into a graph by defining $\alpha$ to be adjacent to $\beta$ if and only if $\alpha \neq \beta$ and $\alpha \cap \beta \neq \emptyset$. Then $G$ operates on $\Gamma$ by right multiplication.

For $\delta \in \Gamma$, let $G_{\delta}=\operatorname{Stab}_{G}(\delta)$, let $G_{\delta}^{(n)}$ equal the largest normal sugroup of $G_{\delta}$ fixing all vertices of distance at most $n$ from $\delta$ and let $\Delta(\delta)$ be the set of all vertices adjacent to $\delta$.

LEMmA 2.10. Let $X$ be any of our groups $\Theta$ or $\Psi, S_{1} \in \operatorname{Syl}_{3}(X)$ and $B_{1}=$ $N_{X}\left(S_{1}\right)$. Then $B_{1}$ is irreducible on $Z\left(S_{1}\right)$; in particular $B_{1}$ is irreducible on $S_{1}$ for $X \cong(P) \mathrm{SL}_{2}(9)$ or $M_{11}$ and $S=Q_{\alpha} Q_{\beta}$.

Proof: See [9].

LEMMA 2.11. The normaliser of a Sylow 3-subgroup is maximal in $\mathrm{SL}_{2}(9)$.

Proof: See [3, 8.3.2 and 11.3.2].

LEMMA 2.12. Let $i=1,2$. Then:

(a) $G_{P_{i} x}=P_{i}^{x}$,

(b) The edge-stabilisers in $G$ are conjugate to $B$,

(c) Let $\delta_{i}=P_{i}$. Then $\Delta\left(\delta_{i}\right) \cong P_{i} / B$ as a $G_{\delta_{i}}$-set; in particular, $G_{\delta_{i}}$ is transitive on $\Delta\left(\delta_{i}\right)$,

(d) Let $(\delta, \lambda)$ be an edge of $\lambda$; then $G=\left\langle G_{\delta}, G_{\lambda}\right\rangle$,

(e) $G$ acts faithfully on $\Gamma$,

(f) $\Gamma$ is connected.

Proof: See $[4,2.1,2.2$ and 3.1] 
Notation 2.13. Let $d($,$) denote the usual distance on the graph \Gamma$. For $\delta \in \Gamma$ and $i \geqslant 1$,

$$
\begin{aligned}
& \Delta^{(i)}(\delta)=\{\lambda \in \Gamma \mid d(\delta, \lambda) \leqslant i\}, \\
& Q_{\delta}=O_{3}\left(G_{\delta}\right), \\
& Z_{\delta}=\left\langle\Omega_{1} Z(T) \mid T \in \operatorname{Syl}_{3}\left(G_{\delta}\right)\right\rangle, \\
& V_{\delta}=\left\langle Z_{\lambda} \mid \lambda \in \Delta(\delta)\right\rangle, \\
& b_{\delta}=\min _{\delta^{\prime} \in \Gamma}\left\{d\left(\delta, \delta^{\prime}\right) \mid Z_{\delta} \not G_{\delta^{\prime}}^{(l)}\right\}, \\
& b=\min _{\delta^{\prime} \in \Gamma}\left\{b_{\delta^{\prime}}\right\} \\
& G_{\delta \lambda}=G_{\delta} \cap G_{\lambda} \text { and } Q_{\delta \lambda}=Q_{\delta} \cap Q_{\lambda} \text { if } \delta \in \Delta(\lambda) .
\end{aligned}
$$

A pair of vertices $\left(\delta, \delta^{\prime}\right)$ such that $Z_{\delta} \not G_{\delta^{\prime}}^{(l)}$ and $d\left(\delta, \delta^{\prime}\right)=b$ is called a critical pair.

The bounding of the parameter $b$ which we just introduced, will allow us to deduce a considerable amount of information about $P_{1}$ and $P_{2}$.

LEMMA 2.14 .

(a) $G$ acts edge- but not vertex-transitively on $\Gamma$,

(b) $G_{\delta}$ is finite,

(c) $C_{G_{\delta}}\left(Q_{\delta}\right) \subseteq Q_{\delta}$,

(d) if $\alpha$ is adjacent to $\beta$ they $\operatorname{Syl}_{3}\left(G_{\alpha} \cap G_{\beta}\right) \subseteq \operatorname{Syl}_{3}\left(G_{\alpha}\right) \cap \operatorname{Syl}_{3}\left(G_{\beta}\right)$.

PROOF: See $[4$, p.73].

REMARK 2.15. Notice that as $G$ acts edge-transitively, $b=\min \left\{b_{\alpha}, b_{\beta}\right\}$ for any pair of adjacent vertices $\alpha, \beta$. Thus, we are allowed to choose $\alpha, \beta$ such that $b_{\alpha}=b \leqslant b_{\beta}$ and $\left\{G_{\alpha}, G_{\beta}\right\}=\left\{P_{1}, P_{2}\right\}$. In particular, $G_{\alpha} \cap G_{\beta}=B$ and $S \in \operatorname{Syl}_{3}\left(G_{\alpha}\right) \cap \operatorname{Syl}_{3}\left(G_{\beta}\right)$.

Let $\alpha \in \Gamma$ be such that $d(\alpha, \alpha)=b$ and $Z_{\alpha} \notin G_{\alpha^{\prime}}^{(l)}$. Let $p$ be a path of length $b$ from $\alpha$ to $\alpha$. We label the vertices of $p$ by

$$
p=(\alpha, \alpha+1, \ldots, \alpha+b)=\left(\alpha^{\prime}-b, \ldots, \alpha^{\prime}-1, \alpha^{\prime}\right),
$$

that is, $\alpha+i$ (respectively $\alpha-i$ ) is the unique vertex in $p$ with $d(\alpha, \alpha+i)=i$ (respectively $d(\alpha-i)=i$ ). furthermore, from 2.12 (c) we may assume that

$$
\beta=\alpha+1 \text { if } b \geqslant 1 .
$$

Note also that if $Q_{\delta}=Q_{\lambda}$ for some $\delta \in \Delta(\lambda)$ then $Q_{\delta} \unlhd\left\langle G_{\delta}, G_{\lambda}\right\rangle=G$, a contradiction. Hence

$$
Q_{\delta} \neq Q_{\lambda} \quad \forall \delta \in \Delta(\lambda) \text {. }
$$

LEMma 2.16. Let $(\delta, \lambda)$ be an edge and let $N$ be a subgroup of $G_{\delta, \lambda}$ such that $N_{G_{\mu}}(N)$ acts transitively on $\Delta(\mu)$ for $\mu \in\{\delta, \lambda\}$. Then $N=1$.

Proof: See $[4,(3.2)]$. 
LEMMA 2.17. For $\delta \in \Gamma$,

(a) $Q_{\delta} \leqslant G_{\delta}^{(1)}$,

(b) $Z_{\delta} \leqslant Z\left(Q_{\delta}\right) \cap V_{\delta}$; in particular, $b \geqslant 1$ and $Z_{\alpha} \leqslant Q_{\alpha^{\prime}}$,

(c) $Z_{\alpha^{\prime}} \leqslant G_{\alpha}$ and $\left[Z_{\alpha}, Z_{\alpha^{\prime}}\right] \leqslant Z_{\alpha} \cap Z_{\alpha^{\prime}}$

(d) $Z_{\alpha} \neq \Omega_{1} Z(T), \quad T \in \operatorname{Syl}_{3}\left(G_{\alpha}\right)$,

(e) If $S \in \operatorname{Syl}_{3}(B)$ and $\Omega_{1}(Z(S))$ is centralised by a sugbroup $R$ of $G_{\beta}$ which acts trasitively on $\Delta(\beta)$ then $Z\left(L_{\alpha}\right)=1$.

Proof: See [9].

REMARK 2.18.

(i) $Z_{\delta} \leqslant G_{\gamma} \forall \gamma \in \Delta^{(1)}(\delta), B=G_{\alpha \beta}, Z_{\alpha} \leqslant B, Z_{\beta} \leqslant B$.

(ii) Also $\operatorname{Syl}_{3}(B) \subseteq \operatorname{Syl}_{3}\left(P_{1}\right) \cap \operatorname{Syl}_{3}\left(P_{2}\right)$.

(iii) A Frattini argument gives that $L_{\delta} S=L_{\delta}$ and for $\mu \in \Delta(\delta), G_{\delta}=L_{\delta} G_{\delta \mu}$.

A list of properties follows, the proofs of which can be found in [9].

LEMMA 2.19.

(i) $\left[Z_{\alpha}, Z_{\alpha^{\prime}}, Z_{\alpha^{\prime}}\right]=1$.

(ii) $V_{\delta} \unlhd G_{\delta} \forall \delta \in \Gamma$.

(iii) $Z_{\alpha}$ normalises $V_{\alpha}$.

(iv) If $\beta>2$ then $V_{\beta}$ is Abelian.

(v) If $Z_{\delta} \leqslant Z\left(L_{\delta}\right)$ then $Z_{\delta} \leqslant Z_{\lambda} \forall \lambda \in \Delta(\delta)$.

(vi) $Z_{\alpha} \notin Z\left(L_{\alpha}\right)$.

(vii) If $Z_{\alpha^{\prime}} \leqslant Z\left(L_{\alpha^{\prime}}\right)$ then $\alpha$ is not conjugate to $\alpha^{\prime}$.

(viii) $Z_{\alpha} \cap Q_{\alpha^{\prime}} \neq C_{Z_{\alpha}}\left(Z_{\alpha^{\prime}}\right)$ if and only if $Z_{\alpha^{\prime}} \leqslant Z\left(L_{\alpha}^{\prime}\right)$.

(ix) Let $\delta \in\{\alpha, \beta\}$ and $A$ be a 3-subgroup of $G_{\delta}$ with $A \& Q_{\delta}$. Then

$$
O^{3}\left(L_{\delta}\right) \leqslant\left\langle A^{L_{\delta}}\right\rangle \text { and } L_{\delta}=\left\langle A^{L_{\delta}}\right\rangle Q_{\delta}
$$

REMARK 2.20 .

(a) By 2.19 (vi), $Z_{\alpha} \& Z\left(L_{\alpha}\right)$ and so by 2.19 (ix) $C_{G_{\alpha}}\left(Z_{\alpha}\right) / Q_{\alpha}$ is a 3-group.

(b) If $Z_{\alpha^{\prime}} \leqslant Q_{\alpha}$ then $\left[Z_{\alpha}, Z_{\alpha^{\prime}}\right]=1$ and so $Z_{\alpha}=C_{Z_{\alpha}}\left(Z_{\alpha^{\prime}}\right)$. Hence $Z_{\alpha} \cap$ $Q_{\alpha^{\prime}} \neq z_{\alpha}$ and $Z_{\alpha} \cap Q_{\alpha^{\prime}} \neq C_{Z_{\alpha}}\left(Z_{\alpha^{\prime}}\right)$.

(c) If $Z_{\alpha^{\prime}} \& Q_{\alpha}$ then by (a) $C_{Z_{\alpha}}\left(Z_{\alpha^{\prime}}\right)=Z_{\alpha} \cap Q_{\alpha^{\prime}}$ and since we have a complete symmetry between $\alpha$ and $\alpha^{\prime}$ in this case, we get that

$$
C_{Z_{\alpha}^{\prime}}\left(Z_{\alpha}\right)=Z_{\alpha^{\prime}} \cap Q_{\alpha}
$$




\section{DEFINITION 2.21:}

(a) $\overline{L_{\delta}}=L_{\delta} / O_{3}\left(L_{\delta}\right)$.

(b) Let $K$ be a complement for $S$ in $B$ and

$$
K_{\alpha}=K \cap L_{\alpha} \text { and } K_{\beta}=K \cap L_{\beta} .
$$

(c) Let $\delta \in\{a, \beta\}$. Let $t_{\delta}$ be an element of order 2 in $K_{\delta}$ with $t_{\delta} Q_{\delta} / Q_{\delta} \in$ $Z\left(L_{\delta} / Q_{\delta}\right)$ if $L_{\delta} / Q_{\delta}$ is isomorphic to one of the groups $\mathrm{SL}_{2}(9), 2 \cdot M_{12}$; otherwise let $t_{\delta}=1$.

The following corollary will be useful for the proof of 3.6.

Corollary 2.22. If $\delta \in \Delta(\lambda), t_{\delta} \neq 1$ and $L_{\lambda} / Q_{\lambda} \cong(P) \mathrm{SL}_{2}(9), M_{11}$ or (2) $M_{12}$ then $t_{\delta}$ does not centralise $S / Q_{\lambda}$.

Proof: See [9].

\section{THE CASE $\left[Z_{\alpha} Z_{\alpha^{\prime}}\right] \neq 1$}

In this section we work under the hypothesis $Z_{\alpha^{\prime}} \nless Q_{\alpha}$. Notice that under this hypothesis, we have a complete symmetry between $\alpha$ and $\alpha^{\prime}$, so $Z_{\alpha^{\prime}} \& Z\left(L_{\alpha^{\prime}}\right)$.

PROPOSITION 3.0. The hypothesis of this section leads to a contradiction.

Lemma 3.1.

(a) $Z_{\alpha} \cap Q_{\alpha^{\prime}}=C_{Z_{\alpha}}\left(Z_{\alpha^{\prime}}\right)$; in particular $b$ is even,

(b) $Z_{\alpha^{\prime}} \cap Q_{\alpha}=C_{\alpha_{\alpha^{\prime}}}\left(Z_{\alpha}\right)$,

Proof: See [9].

DEFinition 3.2: $\varepsilon=1$ if $Z_{\beta} \neq \Omega_{1} Z(S)$ and $\varepsilon=2$ if $Z_{\beta}=\Omega_{1} Z(S)$.

LEMMA 3.3 .

(a) $L_{\alpha} / Q_{\alpha} \cong L_{\alpha^{\prime}} / Q_{\alpha^{\prime}} \cong \mathrm{SL}_{2}$ (9) and $Z_{\alpha}$ is an FF-module for $L_{\alpha} / Q_{\alpha}$.

(b) $Z_{\alpha}=\left[Z_{\alpha}, L_{\alpha}\right] \oplus \Omega_{1} Z\left(L_{\alpha}\right)$ and $\left[Z_{\alpha}, L_{\alpha}\right]$ is the unique natural $\mathrm{SL}_{2}(9)$ module for $L_{\alpha} / Q_{\alpha}$.

Proof: (a) Since $\left[Z_{\alpha}, Z_{\alpha^{\prime}}, Z_{\alpha^{\prime}}\right]=1$ and $\left[Z_{\alpha}, Z_{\alpha^{\prime}}\right] \neq 1$ and as $Z_{\alpha} \notin Q_{\alpha^{\prime}}$ we get that $L_{\alpha^{\prime}} / Q_{\alpha^{\prime}}$ cannot be 3 -stable. Similarly $L_{\alpha} / Q_{\alpha}$ is not 3-stable. Hence

$$
L_{\alpha} / Q_{\alpha} \cong L_{\alpha^{\prime}} / Q_{\alpha^{\prime}} \cong \mathrm{SL}_{2}(9) \text {. }
$$

Without loss of generality we may assume that

$$
\left|Z_{\alpha} Q_{\alpha^{\prime}} / Q_{\alpha^{\prime}}\right| \leqslant\left|Z_{\alpha^{\prime}} Q_{\alpha} / Q_{\alpha}\right|
$$


Let $V=Z_{\alpha}$ and $A=Z_{\alpha^{\prime}} Q_{\alpha} / Q_{\alpha}$. Then

$$
\left|V / C_{V}(A)\right|=\left|Z_{\alpha} / X_{Z_{\alpha}}\left(Z_{\alpha^{\prime}}\right)\right|=\left|Z_{\alpha} / Z_{\alpha} \cap Q_{\alpha^{\prime}}\right|=\left|Z_{\alpha} Q_{\alpha^{\prime}} / Q_{\alpha^{\prime}}\right| \leqslant\left|Z_{\alpha^{\prime}} Q_{\alpha} / Q_{\alpha}\right|=|A|
$$

Therefore $Z_{\alpha}$ is an FF-module for $L_{\alpha} / Q_{\alpha}$.

(b) Follows from 2.8 .

By 3.3, $L_{\alpha}$ fixes some symplectic form on $Z_{\alpha}$ with $\Omega_{1} Z\left(L_{\alpha}\right)$ in its radical. In what follows " $\perp$ " and "singular" is meant with respect to that form on $Z_{\alpha}$ (or also on $\left.Z_{\alpha^{\prime}}\right)$.

LEMMA 3.4. Let $X \leqslant G_{\alpha^{\prime}}$. Then $C_{Z_{\alpha}^{\prime}}(X)^{\perp}=\left[Z_{\alpha^{\prime}}, X\right]+\Omega_{1} Z\left(L_{\alpha^{\prime}}\right)$.

ProOF: See [2, 22.1].

Definition 3.5: Let $\Lambda\left(\alpha, \alpha^{\prime}\right)=\Lambda=\Delta(\alpha) \backslash\{\beta\}$. It is clear that $\Lambda \neq \emptyset$.

LEMMA 3.6. $\varepsilon=2$. In particular $Z_{\alpha}$ is a natural $\mathrm{SL}_{2}(9)$-module and $Z_{\beta} \leqslant Z_{\alpha}$.

Proof: Suppose $\varepsilon=1$. Let $\alpha-1 \in \Lambda$.

If $Z_{\alpha-1} \nless Q_{\alpha^{\prime}-1}$ then $\left(\alpha-1, \alpha^{\prime}-1\right)$ has the same properties as $\left(\alpha, \alpha^{\prime}\right)$, which can't happen as the vertices alternate in terms of 3-stability. Hence

$$
Z_{\alpha-1} \leqslant Q_{\alpha^{\prime}-1} \leqslant G_{\alpha^{\prime}-1}^{(1)} \leqslant G_{\alpha^{\prime}}
$$

and

$$
\left[Z_{\alpha-1}, Z_{\alpha^{\prime}} \cap Q_{\alpha}, Z_{\alpha^{\prime}} \cap Q_{\alpha}\right] \leqslant\left[G_{\alpha^{\prime}}, Z_{\alpha^{\prime}}, Z_{\alpha^{\prime}}\right]=1 .
$$

Now, 3-stability of $G_{\alpha-1}$ implies $\left[Z_{\alpha-1}, Z_{\alpha^{\prime}} \cap Q_{\alpha}\right]=1$ which gives

$$
C_{Z_{\alpha^{\prime}}}\left(Z_{\alpha}\right)=Z_{\alpha^{\prime}} \cap Q_{\alpha} \leqslant C_{Z_{\alpha^{\prime}}}\left(Z_{\alpha-1}\right)
$$

Hence

$$
C_{Z_{\alpha^{\prime}}}\left(Z_{\alpha-1}\right)^{\perp} \leqslant C_{z_{\alpha^{\prime}}}\left(Z_{\alpha}\right)^{\perp}
$$

and by 3.4 ,

$$
\left[Z_{\alpha^{\prime}}, Z_{\alpha-1} \leqslant\left[Z_{\alpha^{\prime}}, Z_{\alpha}\right]\right.
$$

Since $Z_{\alpha-1} Z_{\alpha}$ is normalised by $Z_{\alpha^{\prime}}$ and by $G_{\alpha-1} \cap G_{\alpha}$ we get by choice of $\alpha-1$ that $Z_{\alpha-1} Z_{\alpha} \unlhd G_{\alpha}$ and therefore

$$
C_{G_{\alpha}}\left(Z_{\alpha-1} Z_{\alpha}\right) \unlhd G_{\alpha}
$$

By [9] now, $O^{3}\left(C_{G_{\alpha}}\left(Z_{\alpha-1} Z_{\alpha}\right)\right)=Q_{\alpha} \cap Q_{\alpha-1}$ and so we conclude that

$$
Q_{\alpha-1} \cap Q_{\alpha} \unlhd G_{\alpha} \text { and } Q_{\beta} \cap Q_{\alpha} \unlhd G_{\alpha}
$$


Let $L=\left\langle Q_{\beta}^{G_{\alpha}}\right\rangle$. As $\left[Q_{\beta}, Q_{\alpha}\right] \leqslant Q_{\beta} \cap Q_{\alpha} \unlhd G_{\alpha},\left[L, Q_{\alpha}\right] \leqslant Q_{\alpha} \cap Q_{\beta} \leqslant Q_{\beta}$. Recall the definition of $t_{\alpha}$ (see 2.21) now. Since $Q_{\beta} \not Q_{\alpha} 2.19$ (ix) implies that $t_{\alpha} \in O^{3}\left(L_{\alpha}\right) \leqslant L$. Hence

$$
\left[t_{\alpha}, Q_{\alpha}\right] \leqslant Q_{\alpha} \cap Q_{\beta} \leqslant\left\langle t_{\alpha}\right\rangle\left(Q_{\alpha} \cap Q_{\beta}\right) \unlhd\left\langle t_{\alpha}\right\rangle Q_{\alpha}
$$

and

$$
O^{2}\left(\left\langle t_{\alpha}\right\rangle\left(Q_{\alpha} \cap Q_{\beta}\right)\right) \leqslant\left\langle t_{\alpha}\right\rangle\left(Q_{\alpha} \cap Q_{\beta}\right)
$$

Thus

$$
\left[t_{\alpha}^{S}\right] \leqslant\left\langle t_{\alpha} S\right\rangle \cap Q_{\alpha} \leqslant O^{2}\left(\left\langle t_{\alpha}\right\rangle Q_{\alpha}\right) \cap Q_{\alpha} \leqslant\left(\left\langle t_{\alpha}\right\rangle Q_{\alpha} \cap Q_{\beta}\right) \cap Q_{\alpha} \leqslant Q_{\alpha} \cap Q_{\beta} \leqslant Q_{\beta} .
$$

Hence $t_{\alpha}$ centralises $S / Q_{\beta}$, a contradiction by 2.22 . Thus $\varepsilon=2$. So $Z_{\beta}=\Omega_{1} Z\left(L_{\beta}\right)=$ $\Omega_{1} Z(S)$ and by $2.17(\mathrm{e}), \Omega_{1} Z\left(L_{\alpha}\right)=1$. The last statement of the lemma follows from $3.3(\mathrm{~b})$.

LEMMA 3.7. $Z_{\beta}=C_{Z_{\alpha}}\left(Z_{\alpha^{\prime}}\right)=\left[Z_{\alpha}, Z_{\alpha^{\prime}}\right]+\Omega_{1} Z\left(L_{\alpha}\right)=\left[Z_{\alpha}, Q_{\beta}\right]+\Omega_{1} Z\left(L_{\alpha}\right)=$ $C_{Z_{\alpha}}\left(Q_{\beta}\right)=C_{Z_{\alpha}}(S)$.

Proof: As $\varepsilon=2,\left[Z_{\alpha}, L_{\alpha}\right]$ is 2-dimensional over GF(9). Hence $\left[Z_{\alpha}, L_{\alpha}\right], C_{Z_{\alpha}}\left(Z_{\alpha^{\prime}}\right)$, $\left[Z_{\alpha}, Z_{\alpha^{\prime}}\right],\left[Z_{\alpha}, Q_{\beta}\right]$ and $C_{\left[Z_{\alpha}, L_{\alpha}\right]}\left(Q_{\beta}\right)$ are all 1-dimensional over GF(9). Moreover $\left[Z_{\beta}, Q_{\beta}\right]=1=\left[Z_{\beta}, Z_{\alpha^{\prime}}\right]$ and the lemma follows.

Lemma 3.8. Let $\alpha-1 \in \Lambda$. Then $\left\langle G_{\alpha-1, \alpha}, Z_{\alpha^{\prime}}\right\rangle G_{\alpha}$.

ProOF: Lemma 3.7 implies $\left[Z_{\alpha-1}, Z_{\alpha^{\prime}}\right] \neq 1$ and so $Z_{\alpha^{\prime}} \nless G_{\alpha-1, \alpha}$. By 2.10, $G_{\alpha-1, \alpha}$ is maximal in $G_{\alpha}$ and so $\left\langle G_{\alpha-1, \alpha}, Z_{\alpha^{\prime}}\right\rangle=G_{\alpha}$.

REMARK 3.9. The following are equivalent:

(i) $Z_{\alpha-1}, \not L\left[Z_{\alpha}, Z_{\alpha^{\prime}}\right]$

(ii) $C_{Z_{\alpha-1}}\left(Z_{\alpha^{\prime}}\right)=1$;

Define now $Y_{\beta}^{\bullet}$ and $Y_{\beta}$ by

$$
Y_{\beta}^{\bullet} / Z_{\beta}=\left\langle C_{Z_{\delta} / Z_{\beta}}\left(Q_{\beta}\right) \mid \delta \in \Delta(\beta)\right\rangle
$$

and

$$
Y_{\beta}=C_{Z_{\alpha}}\left(O^{3}\left(L_{\beta}\right)\right)
$$

Note that $\left[Y_{\beta}^{\bullet}, Q_{\beta}\right] \leqslant Z_{\beta}$.

LEMMA $3.10 . b=2$.

PRoof: Suppose $b>2$. Then, by $3.7 C_{Z_{\alpha} / Z_{\beta}}\left(Q_{\beta} / Z_{\beta}\right)=Z_{\alpha}$. As by $[9] Y_{\beta}^{\bullet} \leqslant Z_{\alpha}$ for $b>2$ we get

$$
Z_{\alpha} \leqslant Y_{\beta}^{*} \leqslant Z_{\alpha}
$$


whence

$$
Z_{\alpha}=Y_{\beta}^{\bullet} \unlhd\left\langle G_{\alpha}, G_{\beta}\right\rangle
$$

a contradiction.

Proof of the Proposition: Since $\left[t_{\alpha}, K\right] \leqslant Q_{\alpha} \cap K=1$ we have $\left[t_{\alpha}, K_{\beta}\right]=1$ and the order of $t_{\alpha}$ is 2. By [9], $t_{\alpha}$ induces an inner automorphism on $L_{\beta} / Q_{\beta}$.

By $2.22 t_{\alpha}$ does not centralise $L_{\beta} / Q_{\beta}$. Also, as $t_{\alpha}$ is an inner automorphism we can pick $t \in K_{\beta}$ which acts on the same way on $L_{\beta} / Q_{\beta}$, that is, pick $t \in K_{\beta}$ so that $x_{\beta}=t_{\alpha} t$ and $x_{\beta}$ centralises $L_{\beta} / Q_{\beta}$.

I now claim that the order of $t$ is 2 as well. By choice of $t$,

$$
|t|=\left|t Q_{\alpha} / Q_{\alpha}\right|
$$

and the image of $t$ in $L_{\beta} /\left\langle t_{\beta}\right\rangle Q_{\alpha} \cong L_{\beta}\left\langle x_{\beta}\right\rangle /\left\langle t_{\beta}, x_{\beta}\right\rangle Q_{\alpha}$ is $t_{\alpha}$ which has order two. Hence the claim holds if $t_{\beta}=1$ and so we are done for the cases $\operatorname{PSL}_{2}(9), M_{11}$ or $M_{12}$. The only problem could appear in $2 \cdot M_{12}$ since when we lift $M_{12}$ to $2 \cdot M_{12}$ the order of $t$ could become 4 . But this does not happen by 2.1(b). Moreover in any case $x_{\beta}$ centralises $L_{\beta} / Q_{\beta}$ and the order of $x_{\beta}$ is also one or two.

Now $t_{\alpha}$ acts non-trivially on $Z_{\alpha}$ which is irreducible for $L_{\alpha}$ so $t_{\alpha}$ inverts $Z_{\alpha}$. $K_{\alpha}$ acts on $Y_{\beta}$ faithfully and $K_{\beta}$ centralises $Y_{\beta}$ so $\left[K_{\alpha}, K_{\beta}\right]=1$.

As $Z_{\beta} \leqslant Y_{\beta}$ and $\left|Z_{\beta}^{2}\right|=\left|Z_{\alpha}\right|$ for $L_{\alpha} / Q_{\alpha} \cong \mathrm{SL}_{2}(9)$, we get that $\left|Z_{\alpha}\right| \leqslant\left|Y_{\beta}\right|^{2}$.

$K_{\alpha}$ acts on $Y_{\beta}$ faithfully and $K_{\beta}$ centralises $Y_{\beta}$ so $\left[K_{\alpha}, K_{\beta}\right]=1$. Since $K_{\alpha}$ centralises $t$ and $K_{\alpha}$ centralises $t_{\alpha}$ we get that $K_{\alpha}$ centralises $x_{\beta}$. Thus $\left[x_{\beta}, K_{\alpha}\right]=1$.

Now define $Y_{\beta}=C_{Z_{\alpha}}\left(O^{2}\left(L_{\beta}\right)\right)$. Let $A=Z_{\beta}$.

Since $t$ centralises $Y_{\beta}$ and $t_{\alpha}$ inverts $Y_{\beta}, x_{\beta}$ inverts $Y_{\beta}$ and so $x_{\beta}$ inverts $A$. This means that if $x_{\beta}^{\bullet}$ is the image of $x_{\beta}$ in Aut $(A)$ then $x_{\beta}^{\bullet} \in Z(\operatorname{Aut}(A))$ and so $\left[N_{G_{\alpha}}(A), x_{\beta}^{\bullet}\right]$ centralises $A$.

Let $L=N_{L_{\alpha}}(A)$ and $Q=C_{L_{\alpha}}(A)$. Since $Z_{\alpha}$ is a natural $\mathrm{SL}_{2}(9)$-module, $L / C_{L}(A) \cong G L_{F}(A)$ where $F=G F(9)$ and $L$ acts irreducibly on $A$. Since $A=A^{\perp}$, $\left[Z_{\alpha}, Q\right] \leqslant A^{\perp}=A$. Hence $\left[Z_{\alpha}, Q, Q\right]=1$ and $Q$ is a 3-group. So $Q=O_{3}(L)$. Now $\left[L, x_{\beta}\right] \leqslant Q$ and so by a Frattini argument $L=C_{L}\left(x_{\beta}\right) Q$. Hence $C_{L}\left(x_{\beta}\right)$ acts irreducibly on $A$ and on $Z_{\alpha} / A$ (which is isomorphic to the dual of $A$ ). In particular $x_{\beta}$ inverts or centralises $Z_{\alpha} / A$. Since

$$
V_{\beta}=\left\langle Z_{\alpha}^{G_{\beta}}\right\rangle=\left\langle Z_{\alpha}^{C_{G_{\beta}}\left(x_{\beta}\right)}\right\rangle
$$

we conclude that $x_{\beta}$ inverts or centralises $V_{\beta} / A$.

Note that $x_{\beta}$ inverts $A$ so if $x_{\beta}$ inverts $V_{\beta} / A, x_{\beta}$ inverts $V_{\beta}$ and $V_{\beta}$ is Abelian, a contradiction to $1 \neq\left[Z_{\alpha}, Z_{\alpha^{\prime}}\right] \leqslant V_{\beta}$. 
If $x_{\beta}$ centralises $V_{\beta} / A$ then $V_{\beta}=C_{V_{\beta}}\left(Z_{\beta}\right) A=C_{V_{\beta}}\left(Z_{\beta}\right) \times A$. Hence $V_{\beta}^{\cdot} \leqslant$ $\left(C_{V_{\beta}}\left(Z_{\beta}\right)\right)^{\bullet}$ (as $A \leqslant \bigcap_{g \in G_{\beta}} Z_{\alpha}^{g} \leqslant Z\left(V_{\beta}\right)$ ) and so

$$
V_{\beta^{\prime}}^{\bullet} \cap Z_{\beta} \leqslant\left(C_{V_{\beta}}\left(Z_{\beta}\right)\right)^{\bullet} \cap A=1 .
$$

Hence $C_{V_{\beta^{\prime}}}(S)=1$ and $V_{\beta^{\prime}}=1$, again a contradiction.

\section{THE CASE $\left[Z_{\alpha}, Z_{\alpha^{\prime}}\right]=1$}

In this section we will deal with the case $Z_{\alpha^{\prime}} \leqslant Q_{\alpha}$.

It follows from the hpothesis that there is no symmetry between $\alpha$ and $\alpha^{\prime}$ any more. Also $\left[Z_{\alpha}, Z_{\alpha^{\prime}}\right] \leqslant\left[Z_{\alpha}, Q_{\alpha}\right]=1$ gives

$$
C_{Z_{\alpha}}\left(Z_{\alpha^{\prime}}\right)=Z_{\alpha}
$$

Now notice that $Z_{\alpha} \cap Q_{\alpha^{\prime}} \neq Z_{\alpha}$ (otherwise we get $Z_{\alpha} \leqslant Q_{\alpha^{\prime}}$, a contradiction). Hence, $C_{Z_{\alpha}}\left(Z_{\alpha^{\prime}}\right) \neq Z_{\alpha} \cap Q_{\alpha^{\prime}}$ and by 2.19 (viii), $Z_{\alpha^{\prime}} \leqslant Z\left(L_{\alpha^{\prime}}\right), \alpha$ and $\alpha^{\prime}$ are not conjugate and $b$ is odd. Therefore we have

$$
Z_{\beta}=\Omega_{1} Z\left(L_{\beta}\right) \text { and } Z_{\alpha^{\prime}}=\Omega_{1} Z\left(L_{\alpha^{\prime}}\right) .
$$

LEMMA 4.1. $L_{\alpha^{\prime}} / Q_{\alpha^{\prime}} \cong L_{\beta} / Q_{\beta} \cong \mathrm{SL}_{2}$ (9).

Proof: If $b>1$ then $\left[V_{\alpha^{\prime}}, Z_{\alpha}, Z_{\alpha}\right] \leqslant\left[V_{\alpha^{\prime}}, V_{\beta}, V_{\beta}\right] \leqslant\left[V_{\beta}, V_{\beta}\right]=1$ by 2.19 (iv), so, since $Z_{\alpha} \nless Q_{\alpha^{\prime}}$, we conclude that $L_{\beta} / Q_{\beta}$ is not 3-stable and the claim follows by 2.5 .

If $b=1, Z_{\alpha} \nless Q_{\beta}$ and $\left[Q_{\beta}, Z_{\alpha}, Z_{\alpha}\right]=1$ (by [9]) again imply that $L_{\beta} / Q_{\beta}$ is not 3 -stable and the claim follows by 2.5 .

Notation 4.2. For $\gamma \in \Gamma$ let $D_{\gamma}=C_{Q_{\gamma}}\left(O^{3}\left(L_{\gamma}\right)\right)$.

LemMa 4.3. $Z\left(L_{\alpha}\right)=D_{\alpha}=1$.

ProOF: See [9]

PROPOSITION 4.4. $b=1$.

Proof: Assume that $b>1$. Since $b$ is odd, $b \geqslant 3$.

4.4.1. $V_{\beta}$ has a unique non-central $L_{\beta}$-composition factor; moreover, this composition factor is the natural module for $L_{\beta} / Q_{\beta}$.

Proof: By [9], $\left[V_{\beta} \cap Q_{\alpha^{\prime}}, V_{\alpha^{\prime}}\right]=1$ and hence $V_{\beta} \cap Q_{\alpha^{\prime}} \leqslant C_{V_{\beta}}\left(V_{\alpha^{\prime}}\right)$. By a similar argument we also have that $V_{\alpha^{\prime}} \cap Q_{\beta} \leqslant C_{V_{\alpha^{\prime}}}\left(V_{\beta}\right)$. Without loss of generality, assume

$$
\left|V_{\beta} Q_{\alpha^{\prime}} / Q_{\alpha^{\prime}}\right| \leqslant\left|V_{\alpha^{\prime}} Q_{\beta} / Q_{\beta}\right|
$$


Now let $X=Y / Z$ be a non-central chief factor in $V_{\beta}$. As

$$
C_{Y}\left(V_{\alpha^{\prime}}\right) Z / Z \leqslant C_{Y / Z}\left(V_{\alpha^{\prime}}\right)
$$

we get that

$$
\begin{aligned}
\left|X / C_{X}\left(V_{\alpha^{\prime}}\right)\right| & =\left|Y / Z / C_{Y / Z}\left(V_{\alpha^{\prime}}\right)\right| \leqslant\left|Y / Z / C_{Y}\left(V_{\alpha^{\prime}}\right) Z / Z\right| \\
& =\left|Y / C_{Y}\left(V_{\alpha^{\prime}}\right) Z\right| \leqslant\left|Y / C_{Y}\left(V_{\alpha^{\prime}}\right)\right|=\left|Y / Y \cap C_{V_{\beta}}\left(V_{\alpha^{\prime}}\right)\right| \\
& =\left|Y \cdot C_{V_{\beta}}\left(V_{\alpha^{\prime}}\right) / C_{V_{\beta}}\left(V_{\alpha^{\prime}}\right)\right| \leqslant\left|V_{\beta} / C_{V_{\beta}}\left(V_{\alpha^{\prime}}\right)\right| \\
& \leqslant\left|V_{\beta} Q_{\alpha^{\prime}} / Q_{\alpha^{\prime}}\right| \leqslant\left|V_{\alpha^{\prime}} Q_{\beta} / Q_{\beta}\right|
\end{aligned}
$$

so $X$ is an FF-module; similarly, the direct sum of the $L_{\beta}$ chief factors on $V_{\beta}$ is still an FF-module for $L_{\beta} / Q_{\beta}$ and the lemma follows by 2.6 .

\subsection{2. $\left[V_{\beta}, Q_{\beta}\right] \leqslant D_{\beta}$.}

Proof: Assume that $\left[V_{\beta}, Q_{\beta}\right] \notin D_{\beta}$. Then by 4.4.1, $Z_{\alpha}\left[V_{\beta}, Q_{\beta}\right]$ is normalised by $G_{\alpha \beta} O^{3}\left(L_{\beta}\right)=G_{\beta}$ and we get that $Z_{\alpha}\left[V_{\beta}, Q_{\beta}\right]=V_{\beta}$. Hence $V_{\beta} / Z_{\alpha}=\left[V_{\beta} / Z_{\alpha}, Q_{\beta}\right]$. Since $Q_{\beta}$ is a 3-group acting on the 3-group $V_{\beta} / Z_{\alpha}$ in the above manner, we conclude that $V_{\beta} / Z_{\alpha}=1$. Therefore $V_{\beta}=Z_{\alpha}$, a contradiction. Hence $\left[V_{\beta}, Q_{\beta}\right] \leqslant D_{\beta}$.

4.4.3. Let $Q_{\beta}^{*}=\left[Q_{\beta}, O^{3}\left(L_{\beta}\right)\right]$.

By 4.4.2, $\left[V_{\beta}, Q_{\beta}^{*}\right] \leqslant\left[V_{\beta}, Q_{\beta}\right] \leqslant D_{\beta}$. Note that $Q_{\beta}^{*} \leqslant O^{3}\left(L_{\beta}\right)$ and therefore

$$
\left[V_{\beta}, Q_{\beta}^{*}, Q_{\beta}^{*}\right] \leqslant\left[D_{\beta}, Q_{\beta}^{*}\right] \leqslant\left[D_{\beta}, O^{3}\left(L_{\beta}\right)\right]=1
$$

Hence $\left[Z_{\alpha}, Q_{\beta}^{*}, Q_{\beta}^{*}\right]=1$ and 3 -stability of $L_{\alpha}$ gives that $\left[Z_{\alpha}, Q_{\beta}^{*}\right]=1$ whence $Q_{\beta}^{*} \leqslant Q_{\alpha}$.

\subsubsection{The hypothesis that $b>1$ gives a contradiction.}

Proof: By 4.4.3, $Q_{\beta}^{*}$ centralises $Z_{\alpha}$ and so it centralises $\left\langle Z_{\alpha}^{G_{\beta}}\right\rangle=V_{\beta}$ as well. Since $\left[t_{\beta}, Q_{\beta}\right] \leqslant Q_{\beta}^{*}, t_{\beta}$ is the unique involution in $t_{\beta} Q_{\beta} / Q_{\beta}^{*}$ and so $t_{\beta} Q_{\beta}^{*} \in$ $Z\left(L_{\beta} / Q_{\beta}^{*}\right)$. In particular, $L_{\beta}$ normalises $\left[V_{\beta}, t_{\beta}\right]$. By $4.4 .1,\left[V_{\beta}, t_{\beta}\right] \neq 1$ and so $C_{\left[V_{\beta}, t_{\beta}\right]}(S) \neq 1$. Hence

$$
Z_{\beta} \cap\left[V_{\beta}, t_{\beta}\right] \neq 1
$$

On the other hand, since

$$
V_{\beta}-C_{V_{\beta}}\left(t_{\beta}\right) \times\left[V_{\beta}, t_{\beta}\right]
$$

and $\left[Z_{\beta}, t_{\beta}\right] \leqslant\left[Z_{\beta}, L_{\beta}\right]=1$, we have a contradiction. 
NotATION 4.5. For $\gamma \in \Gamma$ let $F_{\gamma}$ be a normal 3-subgroup of $L_{\gamma}$ minimal with respect to the property $F_{\gamma} \nless D_{\gamma}$.

REMARK 4.6. As $F_{\gamma}$ is a 3-group we get $F_{\gamma} \leqslant Q_{\gamma}$ and $F_{\gamma^{\prime}} \neq F_{\gamma}$. Also, the definition implies $F_{\gamma} \neq 1$. Since $Q_{\gamma}$ is a 3-group acting on the 3-group $F_{\gamma}, F_{\gamma} \neq\left[F_{\gamma}, Q_{\gamma}\right]$ and by minimality of $F_{\gamma},\left[F_{\gamma}, Q_{\gamma}\right] \leqslant D_{\gamma}$. Also it is clear from the definitions that $F_{\beta}=\left[F_{\beta}, O^{3}\left(L_{\beta}\right)\right] \leqslant O^{3}\left(L_{\beta}\right)$ and therefore:

$$
\left[D_{\beta}, F_{\beta}\right] \leqslant\left[D_{\beta}, O^{3}\left(L_{\beta}\right)\right]=1 .
$$

Lemma 4.7. $F_{\beta} \nless Q_{\alpha}$ and $D_{\beta} \leqslant Q_{\alpha}$.

Proof: See [9].

Lemma 4.8. $Q_{\alpha}$ is elementary Abelian, $\left[Q_{\alpha}, O^{3}\left(L_{\alpha}\right)\right]$ is an irreducible $L_{\alpha}$ module and $F_{\alpha}=Z_{\alpha}=\left[Q_{\alpha}, O^{3}\left(L_{\alpha}\right)\right]$. In particular, $\phi\left(D_{\beta}\right)=1$.

Proof: See [9].

COROLlaRY 4.9. $C_{G_{\alpha}}\left(Q_{\alpha}\right)=Q_{\alpha}$. In particular, if $X \leqslant G_{\alpha}$ then $Z \cap Q_{\alpha}=$ $C_{X}\left(Q_{\alpha}\right)$.

Proof: See [9].

Proposition 4.10. $\Theta \not$ (2) $\cdot M_{12}$; in particular, $\Theta \cong \mathrm{PSL}_{2}$ (9) or $M_{11}$.

Proof: By 4.1, $L_{\beta} / Q_{\beta} \cong \mathrm{SL}_{2}(9)$. Also from $Q_{\alpha} Q_{\beta}=S$ we get

$$
\left[F_{\beta} Q_{\alpha} / Q_{\alpha}, S\right]=\left[F_{\beta} Q_{\alpha} / Q_{\alpha}, Q_{\beta}\right]
$$

and as $\left[F_{\beta} Q_{\beta}\right] \leqslant D_{\beta} \leqslant Q_{\alpha}$ (see 4.6 and 4.7 ) 23 conclude that

$$
\left[F_{\beta} Q_{\alpha} / Q_{\alpha}, Q_{\beta}\right]=1
$$

Hence $F_{\beta} Q_{\alpha} / Q_{\alpha} \leqslant Z\left(S / Q_{\alpha}\right)$.

Since $\left|S / Q_{\alpha}\right|=3^{3}$ and $S / Q_{\alpha}$ is not Abelian we get that

$$
\left|Z\left(S / Q_{\alpha}\right)\right|=3
$$

and therefore $F_{\beta} Q_{\alpha} / Q_{\alpha}=Z\left(S / Q_{\alpha}\right)$. But $F_{\beta} \leqslant G_{\alpha}$ and therefore 4.9 gives $\left|F_{\beta} / C_{F_{\beta}}\left(Q_{\alpha}\right)\right|=3$. In particular $F_{\beta} / D_{\beta}$ is an FF-module for $L_{\beta} / Q_{\beta}$. As $L_{\beta} / Q_{\beta} \cong$ $\mathrm{SL}_{2}(9)$, by $2.8, F_{\beta} / D_{\beta}$ is a natural $\mathrm{SL}_{2}(9)$-module, a contradiction to $\left|F_{\beta} / C_{F_{\beta}}\left(Q_{\alpha}\right)\right|=$ 3 .

REMARK 4.11. Since a Sylow 3-subgroup of $\Theta$ is elementary Abelian we have

$$
\Phi\left(Q_{\beta}\right) \leqslant Q_{\alpha} .
$$

Similarly $\Phi\left(Q_{\alpha}\right) \leqslant Q_{\beta}$. 
LEMmA 4.12 . If $N \leqslant S, N \unlhd B, \delta \in\{\alpha, \beta\}$ then $N \leqslant Q_{\delta}$ or $\mathrm{NQ}_{\delta}=S$. In particular, $S=Z_{\alpha} Q_{\beta}$.

ProOF: See [9].

LEMmA 4.13. Let $X_{\beta}=\bigcap_{\delta \in \Delta(\beta)} Q_{\delta}$. Then:

(a) $Q_{\beta} / X_{\beta}$ is an irreducible $G_{\beta}$-module,

(b) $\left[Q_{\beta} / D_{\beta}, t_{\beta}\right]=Q_{\beta} / D_{\beta}$ and $C_{Q_{\beta} / D_{\beta}}=1$,

(c) $C_{Q_{\beta}}\left(t_{\beta}\right) \leqslant D_{\beta}$ and

(d) $X_{\beta}=D_{\beta}$.

Proof: Let $X_{\beta}<A \leqslant Q_{\beta}$ with $A \unlhd G_{\beta}$. Then $A \leqslant Q_{\alpha}$ (since if $A \leqslant Q_{\alpha}$ and $\gamma=\beta^{g}$ with $g \in G_{\beta}$ then since $A \unlhd G_{\beta}$ we get

$$
A+A^{g} \leqslant Q_{\beta}^{g}=Q_{\beta} \mathrm{g}=Q_{\gamma}
$$

which gives $A \leqslant X_{\beta}$, a contradiction). Hence by $8.2, A Q_{\alpha}=S$ and therefore $\left[Z_{\alpha}, Q_{\beta}\right] \leqslant\left[Z_{\alpha}, A\right] \leqslant A$. By $4.11 Q_{\beta^{\prime}} \leqslant X_{\beta}$ and so

$$
\left[L_{\beta}, Q_{\beta}\right]=\left[\left\langle Z_{\alpha}^{G_{\beta}}\right\rangle Q_{\beta}, Q_{\beta}\right] \leqslant A .
$$

Let $\widetilde{\mathcal{Q}}_{\beta}+Q_{\beta} / X_{\beta}$. Then $\widetilde{\mathcal{Q}}_{\beta}$ is Abelian. Now $\widetilde{\mathcal{Q}}=\mathcal{C}_{\widetilde{\mathcal{Q}}_{\beta}}\left(t_{\beta}\right) \times\left[\tilde{\mathcal{Q}}_{\beta}, t_{\beta}\right]$ and both parts are normalised by $L_{\beta}$.

If $\mathcal{C}_{\widetilde{\boldsymbol{Q}}_{\beta}}\left(t_{\beta}\right) \neq 1$, we may assume $A=C_{\mathcal{Q}_{\beta}}\left(t_{\beta}\right) X_{\beta}$ (since then $A \leqslant Q_{\beta}, A \unlhd G_{\beta}$ and as $\mathcal{C}_{\widetilde{\boldsymbol{Q}}_{\beta}}\left(t_{\beta}\right) \neq 1$ we also have $\left.X_{\beta} \neq A\right)$. Hence

$$
\widetilde{A}=\mathcal{C}_{\tilde{Q}_{\beta}}\left(t_{\beta}\right)
$$

and we get $\left[\left[\widetilde{\mathcal{Q}}_{\beta}, t_{\beta}\right], t_{\beta}\right] \leqslant\left[\left[L_{\beta}, Q_{\beta}\right], t_{\beta}\right] \leqslant\left[A, t_{\beta}, t_{\beta}\right]=1$. Hence (element of order 2 acting on a 3 -group) $\left[\widetilde{\mathcal{Q}}_{\beta}, t_{\beta}\right]=1$, a contradiction to $\left[\widetilde{\mathcal{Q}}_{\beta}, Q_{\alpha}, Q_{\alpha}\right]=1$ and the 3-stability of $L_{\beta} /\left\langle t_{\beta} Q_{\beta}\right\rangle$. Therefore $\mathcal{C}_{\widetilde{\mathcal{Q}}_{\beta}}\left(t_{\beta}\right)=1$ and $\widetilde{\mathcal{Q}}_{\beta}=\left[\widetilde{\mathcal{Q}}_{\beta}, t_{\beta}\right]=\left[\widetilde{\mathcal{Q}}_{\beta}, L_{\beta}\right]$. Thus $\widetilde{\mathcal{Q}}_{\beta} \leqslant\left[L_{\beta}, \widetilde{\mathcal{Q}}_{\beta}\right] \leqslant \widetilde{A}$ which implies $\widetilde{A}=\widetilde{\mathcal{Q}}_{\beta}$ and $Q_{\beta} / X_{\beta}$ is an irreducible $G_{\beta}$-module.

Now by $4.7, D_{\beta} \leqslant Q_{\alpha}$ and as $D_{\beta} \unlhd G_{\beta}$ we get $D_{\beta} \leqslant X_{\beta}$. But $\left[X_{\beta}, Z_{\alpha}\right] \leqslant$ $\left[Q_{\alpha}, Z_{\alpha}\right]=1$ and $Z_{\alpha} \notin Q_{\beta}$ give $X_{\beta} \leqslant D_{\beta}$. Hence $X_{\beta}=D_{\beta}$.

LEMMA 4.14. There is $g \in G_{\beta}$ such that $t_{\beta} \in\left\langle Z_{\alpha}, Z_{\alpha}^{g}\right\rangle Q_{\beta}$.

Proof: If $\Psi \cong 2 \cdot A_{5}$ it is clear since in this case

$$
L_{\beta}=\left\langle Z_{\alpha}, Z_{\alpha}^{g}\right\rangle Q_{\beta}
$$

for some $g \in G_{\beta}$ and $t_{\beta} \in L_{\beta}$ by defintion. Since inside $\mathrm{SL}_{2}(9)$ we can generate a $2 \cdot A_{5}$ this case is also clear. 
NOTATION 4.15. $\overline{\mathcal{Q}_{\gamma}}=Q_{\gamma} / D_{\gamma}$.

LEMma 4.16. $\left|\overline{\mathcal{Q}_{\beta}}\right|=3^{4}$.

Proof: By 4.14, pick $g \in G_{\beta}$ such that

$$
t_{\beta} \in\left\langle Z_{\alpha}, Z_{\alpha}^{g}\right\rangle Q_{\beta}
$$

Since $\left|Q_{\beta} / C_{\mathcal{Q}_{\beta}}\left(Z_{\alpha}\right)\right|=\left|Q_{\beta} Q_{\alpha} / Q_{\alpha}\right|+\left|S / Q_{\alpha}\right|=3^{2}$, we get

$$
\left|\overline{\mathcal{Q}_{\beta}} / C_{\overline{\bar{Q}_{\beta}}}\left(t_{\beta}\right)\right| \leqslant 3^{4} .
$$

By $4.13(\mathrm{~b}), C_{\overline{\mathcal{Q}_{\beta}}}\left(t_{\beta}\right)=1$ and therefore $\left|\overline{\mathcal{Q}_{\beta}}\right| \leqslant 3^{4}$. Suppose $\left|\overline{\mathcal{Q}_{\beta}}\right|<3^{4}$. Since 5 does not divide $|\mathrm{GL} 3(3)|$ we conclude that $L_{\beta} / Q_{\beta} \not \mathrm{SL}_{2}(9)$, and contradiction. Hence $\left|\overline{\mathcal{Q}_{\beta}}\right|=3^{4}$.

LEMMA 4.17. $\mid\left[Z_{\alpha}, \overline{\mathcal{Q}_{\beta}}|=| \overline{\mathcal{Q}_{\alpha \beta}}|=| \overline{\mathcal{Q}_{\beta} \cap Z_{\alpha}} \mid=9\right.$.

Proof: If $\left|\left[Z_{\alpha}, \overline{\mathcal{Q}_{\beta}}\right]\right|=3$, then, with same argument as before, we get

$$
\left|\overline{\mathcal{Q}_{\beta}}\right|=\left|\left[\overline{\mathcal{Q}_{\beta}}, t_{\beta}\right]\right| \leqslant 3^{2},
$$

a contradiction. Hence

$$
9 \leqslant \mid\left[Z_{\alpha}, \overline{\mathcal{Q}_{\beta}}|| \leqslant\left|\overline{\mid C Q_{\beta} \cap A_{\alpha}}\right| \leqslant\left|\overline{\mathcal{Q}_{\alpha \beta}}\right| \leqslant 9\right.
$$

and the lemma is proved.

Lemma 4.18. $D_{\beta}=Z_{\beta}$.

Proof: First, show $D_{\beta} \leqslant Z_{\beta}$. Let $L=\left\langle Z_{\alpha}^{G_{\beta}}\right\rangle$. Then by 2.19 (ix), $O^{3}\left(L_{\beta}\right) \leqslant L$ and $L_{\beta}=L Q_{\beta}$. Since $\overline{\mathcal{Q}_{\beta}}$ is irreducible for $G_{\beta}$ we get $\left[\overline{\mathcal{Q}_{\beta}}, L\right]=1$ or $\overline{\mathcal{Q}_{\beta}}$. If $\left[\overline{\mathcal{Q}_{\beta}}, L\right]=1$ then $\left[Q_{\beta}, L\right] \leqslant D_{\beta}$ so $\left[Q_{\beta}, O^{3}\left(L_{\beta}\right)\right]=1$ a contradiction. Therefore $\left[\overline{\mathcal{Q}_{\beta}}, L\right]=\overline{\mathcal{Q}_{\beta}}$ which gives $\left[Q_{\beta}, L\right] D_{\beta}=Q_{\beta}$.

Also, as $L \unlhd G_{\beta}$, we have $Q_{\beta} \leqslant N_{G_{\beta}}(L)$. Hence $\left[Q_{\beta}, L\right] \subseteq L, Q_{\beta} \leqslant D_{\beta} L$ and $L_{\beta}=L D_{\beta}$. But from 4.9 now, $\left[D_{\beta}, D_{\beta}\right] \leqslant \Phi\left(D_{\beta}\right)=1$. As $D_{\beta} \leqslant Q_{\alpha},\left[L, D_{\beta}\right]=1$ so $D_{\beta}$ and $L$ both centralise $D_{\beta}$. But then, we also get $\left[D_{\beta}, L_{\beta}\right]=\left[D_{\beta}, L D_{\beta}\right]=1$. Thus $D_{\beta} \leqslant Z\left(L_{\beta}\right) \leqslant Z_{\beta}$. Therefore $D_{\beta} \leqslant Z_{\beta}$.

Since $Z_{\beta}=\Omega_{1} Z\left(L_{\beta}\right) \leqslant C_{\mathcal{Q}_{\beta}}\left(O^{3}\left(L_{\beta}\right)\right) D_{\beta}$ the lemma follows.

LEMMA 4.19. $Q_{\alpha} \cap Q_{\beta}=Z_{\alpha} \cap Q_{\beta}$.

PROOF: It is enough to show that $Q_{\alpha} \cap Q_{\beta} \leqslant Z_{\alpha} \cap Q_{\beta}$. Let $X \in Q_{\alpha} \cap Q_{\beta}$. Then $x D_{\beta} \in Q_{\alpha} \cap Q_{\beta} / D_{\beta}=\overline{\mathcal{Q}_{\alpha \beta}}=\overline{\mathcal{Q}_{\beta} \cap Z_{\alpha}}=Z_{\alpha} \cap Q_{\beta} / D_{\beta}$. Therefore, $x D_{\beta}=y D_{\beta}$, where $y \in Z_{\alpha} \cap Q_{\beta}$. Then $x=y d, d \in D_{\beta} .2 .19$ (v) gives

$$
Z_{\beta} \leqslant Z_{\alpha}
$$


By $4.18, D_{\beta}=Z_{\beta} \leqslant Z_{\alpha}$. Therefore $x \in Z_{\alpha}$ and hence $x \in Z_{\alpha} \cap Q_{\beta}$.

Corollary 4.20. $Q_{\alpha}=Z_{\alpha}$.

PROOF: Since $Q_{\alpha} \subseteq S=Z_{\alpha} Q_{\beta}$ we get $Q_{\alpha} \subseteq Z_{\alpha} Q_{\beta} \cap Q_{\alpha}=Z_{\alpha}\left(Q_{\alpha} \cap Q_{\beta}\right)$ and hence $Q_{\alpha}=Z_{\alpha}\left(Q_{\alpha} \cap Q_{\beta}\right)=Z_{\alpha}$.

LEMMA 4.21 .

(1) $Q_{\alpha}=Z_{\alpha}$ is irreducible as an $L_{\alpha}$-module

(2) If $\Theta \cong \mathrm{PSL}_{2}$ (9) then $\left|Z_{\alpha}\right|=3^{6},\left|Z_{\beta}\right|=3^{2}$ and $\left|Q_{\beta}\right|=3^{6}$; moreover $\left(L_{\alpha}, L_{\beta}\right) \sim\left(3^{6} \mathrm{PSL}_{2}(9), 3^{1+1+4} \mathrm{SL}_{2}(9)\right)$.

(3) If $\Theta \cong M_{11}$ then $\left|Z_{\alpha}\right|=3^{5},\left|Q_{\beta}\right|=3^{5}$ and $\left|Z_{\beta}\right|=3$; moreover $\left(L_{\alpha}, L_{\beta}\right) \sim\left(3^{5} M_{11}, 3^{1+4} \mathrm{PSL}_{2}(9)\right)$.

ProOF: $2.19(\mathrm{v})$ and 4.18 give $D_{\beta}=Z_{\beta} \leqslant Z_{\alpha}$. Hence

$$
\left|Z_{\alpha} / Z_{\beta}\right|=\left|Z_{\alpha} Q_{\beta} / Q_{\beta}\right|\left|Z_{\alpha} \cap Q_{\beta} / Z_{\alpha} \cap D_{\beta}\right|=\left|Z_{\alpha} Q_{\beta} / Q_{\beta}\right|\left|Z_{\alpha} \cap Q_{\beta} / Z_{\beta}\right|
$$

Recall now 4.17 to get $\left|Z_{\alpha} \cap Q_{\beta} / Z_{\beta}\right|=3^{2}$ and hence

$$
\left|Z_{\alpha} / Z_{\beta}\right|=3^{2}\left|Z_{\alpha} Q_{\beta} / Q_{\beta}\right|=3^{2}\left|S / Q_{\beta}\right|
$$

Since $S / Q_{\beta} \in \operatorname{Syl}_{3}(\Psi)$ we get that

$$
\left|S / Q_{\beta}\right|=3^{2}
$$

Hence $\left|Z_{\alpha} / Z_{\beta}\right| 3^{4}$; in particular, $\left|Z_{\alpha} / Z_{\beta}\right| \leqslant 3^{4}$. Since by [9] we can generate $L_{\alpha}$ by two Sylow 3-subgroups we get $\left|Z_{\alpha}\right| \leqslant 3^{8}$.

By $4.8, Z_{\alpha}$ is irreducible as an $L_{\alpha}$-module.

CASE $\Theta \cong \operatorname{PSL}_{2}(9)$. Then by $2.8\left|Z_{\alpha}\right|=3^{4}$ or $3^{6}$ and since $\left|Z_{\alpha} / Z_{\beta}\right|=3^{4}$ we get that $\left|Z_{\alpha}\right|=3^{6}$ and $\left|Z_{\beta}\right|=3^{2}$.

CASE $\Theta \cong M_{11} \cdot 2.8$ gives that $\left|Z_{\alpha}\right|=3^{5}$ and $\left|Z_{\beta}\right|=3$.

Notice now that in both cases, $D_{\beta}$ is central as by 4.18 we have $D_{\beta}=Z_{\beta}$. Moreover if $\Theta \cong \operatorname{PSL}_{2}(9)$ then $\left|D_{\beta}\right|=3$ and if $\Theta \cong M_{11}$ then $\left|D_{\beta}\right|=3^{2}$. Finally, in both cases, $\left|Q_{\beta} / Z_{\beta}\right|=3^{4}$ and hence $Q_{\beta} / Z_{\beta}$ is an irreducible $L_{\beta}$-module. This completes the proof of the lemma.

ProOF of THEOREM: It follows from 4.10 and 4.21 .

\section{REFERENCES}

[1] J.H. Conway, R.T. Curtis, S.P. Norton, R.A. Parker and R.A. Wilson, Atlas of finite groups (Oxford University Press, Oxford, 1986). 
[2] M. Aschbacher, Finite group theory (Cambridge University Press, Cambridge, 1993).

[3] R.W. Carter, Simple groups of Lie type (John Wiley and Sons Inc., New York, 1989).

[4] A. Delgado and B. Stellmacher, 'Weak (B,N)-pairs of rank 2', in Groups and graphs: new results and methods, (A. Delgado, D. Glodschmidt and B. Stellmacher, Editors) (DMV Seminar Bd 6, Basel, Boston, Stuttgart, 1985).

[5] D. Gorenstein, Finite groups (Chelsea Publishing Co., New York, 1980).

[6] P.J. Greenberg, Mathieu groups (Courant Institute of Mathematical Sciences, New York University, 1973).

[7] G.D. James, 'The modular character of the Mathieu Groups', J. Algebra 27 (1973), 57-111.

[8] U. Meierfrankenfeld, 'Pushing up $S p(4, q)$ ', J. Algebra 112 (1988), 467-477.

[9] P. Papadopoulos, 'Some $\mathrm{SL}_{2}(3)$ amalgams in characteristic 3', Bull. Greek Math. Soc. (to appear).

[10] E. Witt, 'Die 5-fach transitiven Gruppen von Mathieu', Abl. Math. Hamburg 12 (1937), 256-246.

Technological Education Institution of Thesaloniki

Greece 